\title{
SNHG3/miR-148a-3p Axis-mediated High Expression of DNMT1 Correlates with Poor Prognosis and Tumor Immune Infiltration of Hepatocellular Carcinoma
}

\section{Ninghua Yao}

Affiliated Hospital of Nantong University https://orcid.org/0000-0002-7607-2516

Wei Jiang

Wuxi No 2 People's Hospital

Jie Sun

Nantong University Affiliated Hospital: Affiliated Hospital of Nantong University

\section{Chen Yang}

Shanghai Cancer Institute

Wenjie Zheng ( $\nabla$ wenjiezheng@ntu.edu.cn )

Affiliated Hospital of Nantong University https://orcid.org/0000-0002-3073-4596

\section{Yan Wang}

Affiliated Hospital of Nantong University

\section{Research article}

Keywords: DNA-methyltransferase 1, hsa-miR-148a-3p, hepatocellular carcinoma, tumor immune infiltration, noncoding RNA

Posted Date: September 30th, 2021

DOI: https://doi.org/10.21203/rs.3.rs-864636/v1

License: (c) (i) This work is licensed under a Creative Commons Attribution 4.0 International License. Read Full License 
1 SNHG3/miR-148a-3p axis-mediated high expression of DNMT1 correlates with

2 poor prognosis and tumor immune infiltration of hepatocellular carcinoma

3 Ninghua Yao ${ }^{1,2 \#}$, Wei Jiang ${ }^{3 \#}$, Jie Sun ${ }^{1}$, Chen Yang $^{4}$, Weijie Zheng ${ }^{2}$, Yan Wang ${ }^{1}$

$4 \quad{ }^{1}$ Department of Cancer Center, Affiliated Hospital of Nantong University, Nantong

5 226001, Jiangsu, China.

$6{ }^{2}$ Research Center of Clinical Medicine, Affiliated Hospital of Nantong University,

7 Nantong 226001, Jiangsu, China.

$8{ }^{3}$ Department of Neurology, the Second People's Hospital of Wuxi, Wuxi, Jiangsu,

9 China.

$10{ }^{4}$ State Key Laboratory of Oncogenes and Related Genes, Shanghai Cancer Institute,

11 Renji Hospital, Shanghai Jiao Tong University School of Medicine, Shanghai, China.

13 Author contributions:

14 (I) Conception and design: N Yao, W Jiang, and C Yang; (II) Administrative support:

15 Y Wang; (III) Provision of study materials or patients: W Zheng; (IV) Collection and

16 assembly of data: J Sun, and W Jiang; (V) Data analysis and interpretation: W Zheng,

17 and N Yao; (VI) Manuscript writing: All authors; (VII) Final approval of manuscript:

18 All authors. 


\section{Correspondence to:}

21 Y. Wang. Department of Cancer Center, Affiliated Hospital of Nantong University, 20

22 Xisi Road, Nantong 226001, Jiangsu, China. Tel: +8651381161252.

23 E-mail: wang-yan1991@126.com.

24 W. Zheng. Research Center of Clinical Medicine, Affiliated Hospital of Nantong 25 University, Nantong 226001, Jiangsu, China. Tel: +8651385052413.

26 E-mail: wenjiezheng@ntu.edu.cn.

Abstract

\section{Background}

31 Epigenetic reprogramming plays an important role in the occurrence, development, and responsible for maintenance methylation. Nevertheless, the role and mechanism of DNMT1 in HCC remains poorly defined.

\section{Methods}

37 In the current study, we conducted pan-cancer analysis for DNMT1's expression and 38 prognosis using The Cancer Genome Atlas (TCGA) data set. We conducted gene Set 39 Enrichment Analysis (GSEA) between high-and-low DNMT1 expression groups to 
identify DNMT1-related functional significance. We also investigated the relationship

41 between DNMT1 expression and tumor immune microenvironment, including immune cell infiltration and the expression of immune checkpoints. Through a combination series of computer analyses (including expression analyses, correlation analyses, and survival analyses), the noncoding RNAs (ncRNAs) that contribute to the overexpression of DNMT1 were ultimately identified.

\section{Results}

We found that DNMT1 was upregulated in 16 types of human carcinoma including

HCC, and DNMT1 might be a biomarker predicting unfavorable prognosis in HCC patients. DNMT1 mRNA expression was statistically associated with age, histological grade, and the level of serum AFP. Moreover, DNMT1 level was significantly and

51 positively linked to tumor immune cell infiltration, immune cell biomarkers, and

52 immune checkpoint expression. Meanwhile, Gene Set Enrichment Analysis (GSEA)

53 revealed that high-DNMT1 expression was associated with epithelial mesenchymal

54 transition (EMT), $\mathrm{E}_{2} \mathrm{~F}$ target, $\mathrm{G}_{2} \mathrm{M}$ checkpoint, and inflammatory response. Finally, 55 through a combination series of computer analyses the SNHG3/hsa-miR-148a$563 \mathrm{p} / \mathrm{DNMT1}$ axis was confirmed as the potential regulatory pathway in HCC.

\section{Conclusion}

SNHG3/miR-148a-3p axis upregulation of DNMT1 may be related to poor outcome, tumor immune infiltration, and regulated malignant properties in HCC. 
61 Keywords: DNA-methyltransferase 1; hsa-miR-148a-3p; hepatocellular carcinoma; tumor immune infiltration; noncoding RNA

\section{Introduction}

Hepatocellular carcinoma (HCC) is the fourth-leading cause of cancer-associated mortality worldwide (1), with over 100,000 new cases diagnosed and deaths each year (2). In China, the 5-year survival rate for HCC is only $14.1 \%$, and the recurrence rate is about $70 \%$ (3). Chemotherapy, liver transplantation, and surgery are common treatments, but they are only suitable for early-stage HCC patients (4). Since the molecular pathogenesis of HCC remains to be elucidated, it is essential to identify and

71 characterize novel cancer-promoting genes to enable a better understanding this deadly

72 disease, identify promising prognostic biomarkers, and develop more effective clinical 73 therapies. Epigenetic reprogramming regulates the malignant properties of HCC. DNA methylation is a key epigenetic regulatory mechanism that determines the pool of cancer stem cells in liver cancer and possibly other solid tumors (5) that are usually catalyzed by DNA methyltransferases (DNMTs) DNMT1, DNMT3a, and DNMT3b (6).

79 Site-specific hypermethylation and silencing of putative tumor-suppressor genes associated with abnormal expression of DNMTs could cause carcinogenesis and tumor

81 progression (7). DNMT1 is considered to be a maintenance DNA methyltransferase 
82 which mainly maintains $\mathrm{CpG}$ methylation and is involved in embryonic development

83 and somatic cell survival(8). DNMT1 is universally overexpressed in proliferating cells.

84 Extensive studies have indicated that DNMTI is closely associated with tumorigenesis

85 and metastasis in various cancers, including melanoma (9), prostate cancer (10),

86 pancreatic cancer (11), head and neck squamous carcinoma (12), and breast cancer (13).

87 Overexpression of DNMT1 in tumors indicates a poor prognosis (14). Furthermore, it

88 has been reported that DNMT1 could modulate the immune system by maintaining

89 forkhead box P3 (Foxp3) DNA methylation (15). Nevertheless, comprehensive studies

90 on the expression, prognosis, and mechanisms of DNMT1 in HCC remain to be

91 conducted, and the relationship between DNMT1 and tumor immune infiltration in

$92 \quad$ HCC has been poorly defined.

93

94 In the present study, we first conducted expression analyses and survival analyses of

95 DNMT1 in various human cancers. Next, we investigated microRNA (miRNA)-related

96 and long noncoding RNAs (IncRNA)-related DNMT1 regulation in HCC. We

97 ultimately clarified the relationship between DNMT1 expression and tumor immune

98 microenvironment, including immune cell infiltration and the expression of immune

99 checkpoints.

$101 \quad$ Methods 
103 Level 3 RNA-sequencing (RNA-seq) data (Fragments Per Kilobase per Million

$104[\mathrm{FPKM}])$ of 33 types of human cancer, including from The Cancer Genome Atlas-

105 Liver Hepatocellular Carcinoma (TCGA-LIHC) data set and corresponding clinical

106 information, were obtained from TCGA Genomic Data Commons (GDC,

107 https://portal.gdc.cancer.gov/). The RNA-seq data (FPKM values) were then

108 transformed to transcripts-per-million reads (TPM) and normalized into $\log 2(T P M+1)$.

109

110 Kaplan-Meier plotter analysis

111 Survival analysis for DNMT1, including overall survival (OS) and relapse-free survival

112 (RFS) in 8 types of human cancer, were analyzed using the Kaplan-Meier plotter

113 (http://kmplot.com/analysis/), which is an online database able to assess the association

114 of RNA expression with survival in 21 cancer types.

115

116 Gene Expression Profiling Interactive Analysis database analysis

117 The correlation between DNMT1 and biomarkers of immune cells in HCC was

118 analyzed using data from TCGA by Gene Expression Profiling Interactive Analysis

119 (GEPIA, http://gepia.cancer-pku.cn/) (16).

\section{Candidate miRNA prediction and starBase database analysis}

122 Upstream binding miRNAs of DNMT1 were predicted by the following miRNA-target

123 prediction programs: PITA (https://tools4mirs.org/software/target_prediction/pita/), 
RNA22 (https://cm.jefferson.edu/rna22/), miRmap (https://mirmap.ezlab.org/), microT

125 (https://bio.tools/DIANA-microT), miRanda, PicTar (https://pictar.mdc-berlin.de/), 126 and TargetScan (http://www.targetscan.org/vert_72/). We finally only included those 127 miRNAs appearing in 2 or more of the above programs as candidate miRNAs of 128 DNMT1 for subsequent analysis. The expression correlation between candidate 129 miRNAs and DNMT1 and the expression level of hsa-miR-148a-3p in HCC were 130 analyzed with the starBase v3.0 project (http://starbase.sysu.edu.cn/). In addition, 131 starBase was employed to predict candidate lncRNAs potentially binding to hsa-miR132 148a-3p. The expression correlation between hsa-miR-148a-3p and SNHG3 or between 133 SNHG3 and DNMT1 in HCC were also analyzed by starBase.

\section{Gene Set Enrichment Analysis}

136 Gene Set Enrichment Analysis (GSEA, http://software.broadinstitute.org/gsea/index.

137 jsp) was conducted between high-and-low DNMT1 expression groups to identify 138 DNMT1-related functional significance based on the Hallmark gene set 139 ("h.all.v7.0.symbols.gmt”). Statistical significance was considered at $|\mathrm{NES}|>1$, adjusted $140 P$ value $<0.05$, and false discovery rate $($ FDR $)<0.05$.

\section{Cell composition fraction estimation}

143 To make reliable immune infiltration estimations, we used "immunedeconv"

144 (https://icbi-lab.github.io/immunedeconv/), an R package that integrates 6 state-of- 

quanTIseq. Visualization was performed using the software packages "ggplot2" and "pheatmap".

\section{Additional bioinformatic and statistical analysis}

We used R software (version 3.6.3, https://www.r-project.org/) to analyze data and plot graphs. Wilcoxon rank-sum test for unpaired samples and the Wilcoxon signed-rank test was used for paired samples. Visualization was performed using the R package "ggplot2" (http://ggplot2.org). Patient characteristics between groups were compared using the chi-square test and Fisher's exact test. Correlations of all RNAs and those

155 between the levels of RNAs and immune checkpoints in HCC were analyzed using

156 Spearman's correlation and visualized using "ggplot2". Survival analysis for all RNAs

157 in the competing endogenous RNA (ceRNA) network was carried out and visualized

158 using the survival package in R (https://CRAN.R-project.org/package=survival). The

159 differentially expressed genes (DEGs) were determined by limma tests with $160|\log 2(\mathrm{FC})|>1$ and adjusted $P$ value $<0.05(17)$. The Kruskal-Wallis test was used to 161 analyze the RNA expression among different histological grades. A $P$ value $<0.05$ was 162 considered statistically significant.

\section{Results}


We first analyzed the expression of DNMT1 in 33 types of TCGA-ALL (normal $=730$, tumor $=11,363)$ database to investigate its possible roles in carcinogenesis. As presented in Fig. 1A, DNMT1 was upregulated in 16 types of human carcinoma, 169 including bladder cancer (BLCA), cervical and endocervical cancer (CESC), breast cancer (BRCA), cholangiocarcinoma (CHOL), colon adenocarcinoma (COAD), 171 esophageal carcinoma (ESCA), head and neck squamous cell carcinoma (HNSC), 172 glioblastoma multiforme (GBM), kidney renal clear cell carcinoma (KIRC), LIHC, 173 lung adenocarcinoma (LUAD), pheochromocytoma and paranglioma (PCPG), rectum 174 adenocarcinoma (READ), stomach adenocarcinoma (STAD), thyroid carcinoma 175 (THCA), and uterine corpus endometrial carcinoma (UCEC), and downregulated in 176 kidney chromophobe $(\mathrm{KICH})$, while not significantly altered in kidney renal papillary 177 cell carcinoma (KIRP), pancreatic adenocarcinoma (PAAD), thyoma (THYM), or 178 prostate adenocarcinoma (PRAD), compared with corresponding normal tissue. Next, 179 we verified the expression of DNMT1 in TCGA tumor tissues compared with paired 180 normal tissues. We found that the expression of DNMT1 was statistically increased in 181 UCEC, LIHC, BRCA, ESCA, HNSC, STAD, READ, BLCA, KIRC, LUAD, and LUSC 182 and significantly reduced in THCA (Fig. 1B-1M). In summary, DNMT1 was 183 upregulated in UCEC, LIHC, BRCA, ESCA, HNSC, STAD, READ, BLCA, KIRC, 184 and LUAD, indicating that DNMT1 might play a key regulatory role in the 185 carcinogenesis of these 10 cancers. 
188 We next performed survival analysis, including OS and RFS, for DNMT1 in UCEC,

189 BLCA, BRCA, HNSC, LIHC, LUSC, THCA, and LUAD. For OS, LIHC patients

190 with higher expression of DNMT1 had a poorer prognosis; however, those with a

191 higher expression with HNSC had a better prognosis (Fig.2). For RFS, increased

192 expression of DNMT1 was correlated with poor clinical outcomes in LIHC and

193 THCA patients (Fig. 3). Taken together, these data suggested that DNMT1 could be

194 used as a biomarker predicting unfavorable prognosis in HCC patients.

195

Relationship between DNMT1 expression and clinical characteristics in HCC

197 We downloaded clinical and gene expression data of HCC patients from TCGA

198 database, including gender, age, histologic grade, alpha-fetoprotein (AFP), OS, Child-

199 Pugh grade, T classification, and pathologic stage. Then, associations between clinical

200 characteristics and DNMT1 mRNA expression in HCC were analyzed. Patients were

201 divided into high- and low-expression groups on the basis of median DNMT1 mRNA

202 expression. Our results revealed that DNMT1 mRNA expression was statistically

203 associated with age, histological grade, and the level of serum AFP (all $P<0.001$,; Table

204 1). 
ncRNAs are responsible for the regulation of gene expression and can be classified into miRNAs, small nucleolar RNAs (snoRNAs), circular RNAs (circRNAs), and lncRNAs (18). To determine whether DNMT1 was regulated by some ncRNAs, we first predicted

210 the upstream miRNAs that might bind to DNMT1 and finally found 14 miRNAs. For

211 better visualization, we established an miRNA-DNMT1 regulatory network using 212 Cytoscape software (https://cytoscape.org/) (Fig.4A). In theory, there should be a 213 negative correlation between miRNA and DNMT1 due to the action mechanism of 214 miRNA. Therefore, we performed expression correlation analysis for miRNA-DNMT1 215 pairs. As shown in Fig. 4B, DNMT1 was significantly and negatively associated with 216 hsa-miR-148a-3p and positively linked to the other 13 predicted miRNAs in HCC. Then, 217 we assessed the expression of hsa-miR-148a-3p in HCC and normal control samples 218 with starBase and evaluated the prognostic value of hsa-miR-148a-3p in HCC with a 219 Kaplan-Meier plotter. As shown in Fig. 4C and 4D, hsa-miR-148a-3p was significantly 220 downregulated in $\mathrm{HCC}$, and its downregulation was correlated with poor prognosis. 221 Together, these findings suggest that hsa-miR-148a-3p might be the most influential 222 upstream miRNA of DNMT1 in HCC.

\section{Prediction and analysis of upstream IncRNAs of hsa-miR-148a-3p}

225 Next, we predicted the upstream lncRNAs of hsa-miR-148a-3p using the starBase 226 database and obtained a total of 45 possible lncRNAs. Similarly, for better visualization, 227 the lncRNA-hsa-miR-148a-3p regulatory network was established using Cytoscape 
229 and normal tissues using the "limma" R package. A Venn diagram was finally created

230 showing 3 overlaps of the 1454 DEGs and 45 possible lncRNAs (Fig. 5B), indicating

231 that LINC01554, small nucleolar RNA host gene 3 (SNHG3), and H19 were the co-

232 expressed differential lnRNAs found in both cohorts. As is presented in the volcano

233 plot in Fig. 5C, SNHG3 was the upregulated gene and LINC01554 or H19 was the

234 downregulated gene in HCC. Subsequently, the prognostic values of the SNHG3 in

235 HCC were assessed, which revealed that overexpressed SNHG3 indicated poor OS of

236 patients with HCC (Fig. 5D). Based on the above results, we next explored whether

237 SNHG3 could regulate hsa-miR-148a-3p expression as a ceRNA in HCC. According

238 to the ceRNA hypothesis, IncRNAs usually serve as ceRNAs by binding to miRNAs,

239 and the key qualified lncRNAs in the ceRNA subnet should be negatively associated

240 with miRNA and positively linked to mRNA at the same time. The expression

241 correlation between SNHG3 and hsa-miR-148a-3p or DNMT1 in HCC is shown in Fig.

242 5E-F. Furthermore, different expressions of DNMT1, has-miR-148a-3p, and SNHG3

243 were observed in normal and HCC tissues of different histologic grades (Fig. 5 G-I).

244 While the expression of DNMT1 and SNHG3 in HCC was statistically increased, the 245 expression of hsa-miR-148a-3p was significantly reduced compared with 246 corresponding normal tissue. Likewise, while high-grade groups (G2/G3/G4) had 247 significantly higher DNMT1 and SNHG3 expression than low-grade groups (G1), the 248 expression of hsa-miR-148a-3p in low-grade groups (G1) was higher. Cumulatively, 
these findings suggested that SNHG3 might serve as a ceRNA to mediate DNMT1 by competitively binding to hsa-miR-148a-3p.

\section{DNMT1 expression was positively related to immune cell infiltration in HCC}

253 Tumor-infiltrating immune cells are independent predictors of cancer survival. As 254 presented in Fig. 6A, there was no significant change in the level of immune cell 255 infiltration with copy number alteration of DNMT1 in HCC. Thus, we conducted a correlation analysis between DNMT1 expression and immune cell infiltration in HCC using TIMER (http://timer.cistrome.org/). The expression of DNMT1 was significantly

258 and positively related to all analyzed immune cells, including $\mathrm{B}$ cell $259(\mathrm{Cor}=0.486 ; P=8.03 \mathrm{e}-22), \mathrm{CD} 8+\mathrm{T}$ cell $(\mathrm{Cor}=0.331 ; P=3.36 \mathrm{e}-10), \mathrm{CD} 4+\mathrm{T}$ cell $260($ Cor $=0.494 ; P=1.40 \mathrm{e}-22)$, macrophage $($ Cor $=0.541 ; P=2.27 \mathrm{e}-24)$, neutrophil $261($ Cor $=0.467 ; P=4.67 \mathrm{e}-20)$, and dendritic cell $(\mathrm{DC}, \mathrm{Cor}=0.536 ; P=1.22 \mathrm{e}-26)$ in 262 HCC (Fig. 6B-G). Then, to further investigate the role of DNMT1 in tumor immunity, 263 HCC patients from TCGA were divided into DMNT1-high and DNMT1-low subgroups.

264 We used XCell (https://xcell.ucsf.edu/) to compare the differences in the abundance of 265 tumor-infiltrating immune cells and extracellular matrix cells between the 2 groups (Fig. 266 S1). Results illustrated that the stromal score was higher in the low-DNMT1 expression 267 group than in the high-DNMT1 expression group $(P<0.001)$. The high-DNMT1 268 expression group had a significantly higher abundance of CD4+ memory T cells, Th2 269 cells, gamma delta T cells (Tgd cells), natural killer (NK) T cells, monocytes, and most 
DC and B cells, but a significantly lower abundance of M2 macrophages, CD4 central

271 memory T cells, CD8+ naive T cells, hematopoietic stem cells (HSCs), granulocyte272 macrophage progenitor (GMP) cells, and endothelial cells compared to the low273 DNMT1 expression group $(P<0.05)$. Finally, we used the GEPIA database to 274 determine the expression correlation of DNMT1 with immune cell biomarkers in HCC. 275 It was revealed that DNMT1 expression had significantly positive correlation with the 276 gene markers of B cells (CD19 and CD79A), CD8+ T cells (CD8A and CD8B), CD4+ 277 T cells (CD4), M1 macrophages (IRF5 and PTGS2), M2 macrophages (CD163, VSIG4, 278 and MS4A4A), neutrophils (ITGAM and CCR7), and DCs (HLA-DPB1, HLA-DRA, 279 HLA-DPA1, CD1C, NRP1, and ITGAX) in HCC. All these results indicated that 280 DNMT1 is positively related to immune cell infiltration.

DNMT1 expression was positively associated with immune checkpoints in HCC

283 Programmed cell death protein 1 (PD-1; also known as PDCD1), programmed death 284 ligand 1 (PD-L1; also known as CD274), and cytotoxic T lymphocyte antigen 4 285 (CTLA4) are important biomarkers of $\mathrm{T}$ cell exhaustion (19). Tumors can escape 286 immune surveillance by taking advantage of immune checkpoints. We plotted the 287 relationship of DNMT1 and PD-1, PD-L1, and CTLA-4 to clarify the relationship 288 between DNMT1 and tumor immune escape. Expression of DNMT1 correlated 289 positively with all 3 of these immune checkpoints, showing statistical significance in 290 HCC (Fig.7A,7C,7E). Similar to GEPIA database analysis, we also found that DNMT1 
291 expression was positively related to that of PD-1, PD-L1, and CTLA4 in HCC, with 292 statistical significance indicated with TIMER (Fig. 7B,7D,7F). These findings 293 confirmed that the carcinogenic effects mediated by DNMT1 might be related to the 294 dysfunctional state of T cells and tumor immune escape.

\section{GSEA identified DNMT1-related hallmark pathways}

297 To determine the potential function of DNMT1 in HCC, GSEA analyses were 298 conducted between the high- and low-DNMT1 expression groups. The top 4 hallmark 299 items (adjusted $P$ value <0.05) involved in the high-DNMT1 expression group were 300 epithelial-mesenchymal transition, $\mathrm{G}_{2} / \mathrm{M}$ checkpoint, E2F_targets, and inflammatory 301 response (Fig. 8A), while the top 4 Hallmark items (adjusted $P$ value $<0.05$ ) involved 302 in the low-DNMT1 expression group were bile acid metabolism, fatty acid metabolism, 303 oxidative phosphorylation, and xenobiotic metabolism (Fig. 8B). Cyclin-dependent 304 kinase 1 (CDK1) is the key regulator of the $\mathrm{G}_{2} / \mathrm{M}$ checkpoint (20). We further analyzed 305 the expression correlation of DNMT1 with CDK1 and E2Fs (E2F1, E2F2, E2F3, E2F4, 306 E2F5, E2F6, E2F7, and E2F8) in HCC. As shown in Fig. 8C, DNMT1 expression was 307 significantly and positively correlated with CDK1 expression. Fig. 8D-8K, further 308 shows that DNMT1 expression was positively related to that of E2F1, E2F2, E2F3, 309 E2F4, E2F5, E2F6, E2F7, and E2F8 in HCC, with statistical significance. 
312 The occurrence and development of HCC is a complex, dynamic biological process that

313 involves genetic, epigenetic cell state, and microenvironment alterations. Clarifying the

314 molecular mechanism underlying HCC carcinogenesis may contribute to the

315 development of effective therapeutic targets or valuable prognostic biomarkers.

316 Accumulating evidence has shown that DNMT1 participates in the tumorigenesis and

317 progression of various human cancers, including HCC. However, to date, knowledge

318 about DNMT1 in HCC remains insufficient, and further research is needed.

320 This study was performed to identify the feasibility of DNMT1 as a promising

321 biomarker in HCC patients. We first performed pan-cancer analysis on the expression

322 of DNMT1 using TCGA database. Then, the survival analysis of DNMT1 in some of

323 the cancer types with statistical significance as analyzed above was conducted,

324 indicating increased expression of DNMT1 to be correlated with poor clinical outcomes

325 in HCC. Moreover, high DNMT1 expression was associated with histological grade.

326 This suggests that upregulated DNMT1 might be involved in malignant transformation,

327 which is in line with previously described results (14).

329 The ceRNA hypothesis consists of lncRNA mainly regulating mRNA through the 330 ceRNA regulatory mechanism and RNAs affecting each other's levels by competing 331 with a limited pool of miRNAs (21). It has been shown that DNMT1 can inhibit the 332 transcription of tumor-suppressive miRNAs in cancer progression by maintaining their 
333 hypermethylation (22). In our study, we developed a lncRNA-miRNA-mRNA triple

334 regulatory network related to DNMT1 in HCC. Through candidate miRNA prediction

335 conducted by the prediction programs-PITA, RNA22, miRmap, microT, miRanda,

336 PicTar, and TargetScan — and correlation analysis - including expression analysis and

337 survival analysis - we finally identified has-miR-148a-3p as the most likely potential

338 upstream miRNA of DNMT1 in HCC. Has-miR-148a-3p is a member of the miR-

$339148 / 152$ family and has been reported to be a tumor suppressor for various human

340 cancers, including pancreatic cancer (23), esophageal cancer (24), and HCC (25).

341 Recent evidence has confirmed that the reciprocal negative regulation between hsa-

342 miR-148a-3p and DNMT1 contributes to cell proliferation, cell cycle processes, and

343 maintaining cell stemness characteristics in HCC (26).

345 Next, upstream lncRNAs of has-miR-148a-3p/DNMT1 axis were also predicted, and

34645 possible lncRNAs were found. We intersected these lncRNAs with 1548 DEGs

347 between TCGA-LIHC tumor samples and normal tissues to screen for differentially

348 expressed lncRNAs. In the end, on the basis of the volcano map, ceRNA hypothesis,

349 and correlation analyses, we identified SNHG3 as the upstream lncRNA. It has been

350 reported that SNHG3 is an oncogene in many kinds of malignancies (27). Meanwhile,

351 multiple miRNAs in HCC have been shown to promote tumor growth and metastasis

352 through targeting SNHG3 (28, 29). Considering these findings, we identified

353 SNHG3/hsa-miR-148a-3p/DNMT1 axis as the potential regulatory pathway in HCC. 
355 At present, compared with other tumors, immunotherapy for liver cancer is still in its 356 infancy (30). Increased infiltration of immune cells in tumors and high expression of 357 immune checkpoints contribute to the efficacy of immunotherapy (31). Epigenetic 358 modulation could enhance immunotherapy for HCC by upregulating previously 359 repressed neoantigens and increasing cytotoxic T-cell infiltration in the 360 immunosuppressive tumor microenvironment (32). It was found that increased 361 methylation of T-cells is beneficial and that epigenetic control in intratumoral T-cells 362 of Foxp3 can regulate the growth of HCC (33). Our results ultimately revealed DNMT1 363 expression to be positively associated with immune cell infiltrates, such as DCs, CD4+ 364 T cells, and B cells. Meanwhile, we also found positive correlations between DNMT1 365 expression and immune checkpoints, which affect T-cell exhaustion and immune 366 escape. Taken together these findings indicate that DNMT1 may be a valuable 367 immunotherapy biomarker, and targeting DNMT1 might enhance the efficacy of 368 immunotherapy in HCC.

370 To further explore the biological functions of DNMT1, we conducted GSEA analyses 371 between the high- and low-DNMT1 expression groups. The GSEA found that Hallmark 372 gene sets enriched in the high-DNMT1 expression group were mainly related to 373 epithelial-mesenchymal transition (EMT), E2F target, $\mathrm{G}_{2} \mathrm{M}$ checkpoint, and 374 inflammatory response. The EMT process is critical for epithelial cell invasion, 
resistance to apoptosis, tumor dissemination, and drug resistance (34). It might be that DNMT1-induced epigenetic silencing of SFRP1 causes activation of the Wnt signaling

377 pathway and increases the aggressiveness of HCC by induction of EMT (35). Both the $378 \mathrm{E} 2 \mathrm{~F}$ and $\mathrm{G}_{2} / \mathrm{M}$ checkpoints are targets associated with the cell cycle. Additionally, E2F 379 activators regulate the transition from the G1 to $\mathrm{S}$ phase in the cell cycle and control 380 cell apoptosis and differentiation (36). The key member of the Hallmark $\mathrm{G}_{2} \mathrm{M}$ 381 checkpoint gene set is CDK1. Meanwhile, we identified that DNMT1 significantly 382 increased the expression of E2Fs and CDK1. These results amply demonstrated that 383 increased DNMT1 participates in tumor progression via deleterious interaction with 384 cell cycle-related molecules. The GSEA analyses also demonstrated enrichment of 385 genes involved in the inflammatory response, which might account for the increased 386 infiltration of immune cells in tumors. Cumulative, these findings elucidate the way in 387 which DNMT1 participates in HCC, which will help future targeted therapy research.

\section{Conclusions}

390 In summary, we established the SNHG3/hsa-miR-148a-3p/DNMT1 axis as the 391 potential regulatory pathway of hepatocarcinogenesis, which was also identified as a 392 biomarker of poor prognosis. We further found that DNMT1 might exert its oncogenic 393 effect through modulating cell cycle progression by regulating transcription and 394 increasing tumor immune cell infiltration and immune checkpoint expression in HCC. 395 However, these results should be validated by more basic hepatocarcinogenesis-related 
experiments in the future.

\section{Declarations}

399 Ethics approval and consent to participate: The authors are accountable for all

400 aspects of the work in ensuring that questions related to the accuracy or integrity of any

401 part of the work are appropriately investigated and resolved.

402 Consent for publication: Not applicable.

403 Availability of data and materia: TCGA Data Poral: https://portal.gdc.cancer.gov/

404 Competing interests: The authors have no conflicts of interest to declare.

405 Funding: This study was supported by grants from the National Natural Science

406 Foundation (no. 82070622 and 81702419), the Key Research and Development Plan of 407 Jiangsu Province (no. BE2020668), and the Nantong Science 389 and Technology 408 Project (no. MS12019013 and MSZ20207).

409 Author contributions: (I) Conception and design: N Yao, W Jiang, and C Yang; (II) 410 Administrative support: Y Wang; (III) Provision of study materials or patients: W 411 Zheng; (IV) Collection and assembly of data: J Sun, and W Jiang; (V) Data analysis 412 and interpretation: W Zheng, and N Yao; (VI) Manuscript writing: All authors; (VII)

413 Final approval of manuscript: All authors.

414 Acknowledgments: We thank the patients and investigators who participated in TCGA

415 for providing the data. 


\section{References}

418 1. Akinyemiju T, et al. (2017) The Burden of Primary Liver Cancer and 419 Underlying Etiologies From 1990 to 2015 at the Global, Regional, and National 420 Level: Results From the Global Burden of Disease Study 2015. JAMA oncology $421 \quad$ 3: $1683-1691$.

422 2. Bray F, et al. (2018) Global cancer statistics 2018: GLOBOCAN estimates of 423 incidence and mortality worldwide for 36 cancers in 185 countries. $C A$ : $a$ cancer journal for clinicians 68: 394-424.

3. Allemani C, et al. (2018) Global surveillance of trends in cancer survival 200014 (CONCORD-3): analysis of individual records for 37513025 patients diagnosed with one of 18 cancers from 322 population-based registries in 71 countries. Lancet (London, England) 391: 1023-1075.

4. Forner A, Reig M, Bruix J. (2018) Hepatocellular carcinoma. Lancet (London, England) 391: 1301-1314.

431 5. Raggi C, et al. (2014) Epigenetic reprogramming modulates malignant 432 properties of human liver cancer. Hepatology (Baltimore, Md.) 59: 2251-2262. 433 6. Jeltsch A, Jurkowska RZ. (2016) Allosteric control of mammalian DNA 434 methyltransferases - a new regulatory paradigm. Nucleic acids research 44: 8556-8575.

436 7. Arechederra M, Daian F. (2018) Hypermethylation of gene body CpG islands 437 predicts high dosage of functional oncogenes in liver cancer 9: 3164.

438 8. Iwanami N, et al. (2020) Transgenerational inheritance of impaired larval T cell 439 development in zebrafish 11: 4505.

440 9. Maric H, et al. (2019) DNMT1 and DNMT3B genetic polymorphisms affect the 441 clinical course and outcome of melanoma patients. Melanoma research 29: 596$442 \quad 602$.

443 10. Lee E, et al. (2016) DNMT1 Regulates Epithelial-Mesenchymal Transition and 444 Cancer Stem Cells, Which Promotes Prostate Cancer Metastasis. Neoplasia 
(New York, N.Y.) 18: 553-566.

446 11. Wong KK. (2020) DNMT1 as a therapeutic target in pancreatic cancer: mechanisms and clinical implications. Cellular oncology (Dordrecht) 43: 779792.

12. Cui J, Zheng L, Zhang Y, Xue M. (2021) Bioinformatics analysis of DNMT1 expression and its role in head and neck squamous cell carcinoma prognosis. Scientific reports 11: 2267.

13. Vernier M, McGuirk S, Dufour CR, Wan L, Audet-Walsh E. (2020) Inhibition

14. Saito Y, et al. (2003) Increased protein expression of DNA methyltransferase of DNMT1 and ERR $\alpha$ crosstalk suppresses breast cancer via derepression of IRF4 39: 6406-6420.

16. Tang Z, et al. (2017) GEPIA: a web server for cancer and normal gene expression profiling and interactive analyses. Nucleic acids research 45: W98-

17. Ritchie ME, et al. (2015) limma powers differential expression analyses for

18. Wang J, et al. (2019) ncRNA-Encoded Peptides or Proteins and Cancer. Molecular therapy : the journal of the American Society of Gene Therapy 27: 1718-1725.

19. Wherry EJ, Kurachi M. (2015) Molecular and cellular insights into T cell exhaustion. Nature reviews. Immunology 15: 486-499.

20. Liu S, et al. (2020) The Severe Fever with Thrombocytopenia Syndrome Virus 
NSs Protein Interacts with CDK1 To Induce G(2) Cell Cycle Arrest and Positively Regulate Viral Replication. Journal of virology 94.

21. Salmena L, Poliseno L, Tay Y, Kats L, Pandolfi PP. (2011) A ceRNA hypothesis: the Rosetta Stone of a hidden RNA language? Cell 146: 353-358.

22. Ning X, et al. (2015) DNMT1 and EZH2 mediated methylation silences the microRNA-200b/a/429 gene and promotes tumor progression. Cancer Lett 359: 198-205.

23. Fu X, et al. (2020) MicroRNA-148a-3p suppresses epithelial-to-mesenchymal transition and stemness properties via Wnt1-mediated $\mathrm{Wnt} / \beta$-catenin pathway in pancreatic cancer. J Cell Mol Med 24: 13020-13035.

24. Wang Y, Hu Y, Guo J, Wang L. (2019) miR-148a-3p Suppresses the Proliferation and Invasion of Esophageal Cancer by Targeting DNMT1. Genetic testing and molecular biomarkers 23: 98-104.

25. Jung KH, et al. (2016) Differentiation therapy for hepatocellular carcinoma: Multifaceted effects of miR-148a on tumor growth and phenotype and liver fibrosis. Hepatology 63: 864-879.

26. Li X, et al. (2020) Casticin inhibits stemness of hepatocellular carcinoma cells via disrupting the reciprocal negative regulation between DNMT1 and miR148a-3p. Toxicology and applied pharmacology 396: 114998.

27. Xu B, et al. (2020) LncRNA SNHG3, a potential oncogene in human cancers 20: 536 .

28. Zhao Q, et al. (2019) LncRNA SNHG3 Promotes Hepatocellular Tumorigenesis by Targeting miR-326. The Tohoku journal of experimental medicine 249: 4356.

29. Zhang PF, et al. (2019) LncRNA SNHG3 induces EMT and sorafenib resistance by modulating the miR-128/CD151 pathway in hepatocellular carcinoma 234: 2788-2794.

30. Johnston MP, Khakoo SI. (2019) Immunotherapy for hepatocellular carcinoma: 
502 31. Yang Y. (2015) Cancer immunotherapy: harnessing the immune system to battle cancer. The Journal of clinical investigation 125: 3335-3337.

504 32. Hong YK, et al. (2019) Epigenetic modulation enhances immunotherapy for

33. Liu Q, et al. (2019) Epigenetic control of Foxp3 in intratumoral T-cells regulates

34. Kahlert UD, Nikkhah G, Maciaczyk J. (2013) Epithelial-to-mesenchymal(-like) transition as a relevant molecular event in malignant gliomas. Cancer Lett 331:

35. Quan H, et al. (2014) Hepatitis C virus core protein epigenetically silences SFRP1 and enhances HCC aggressiveness by inducing epithelial-mesenchymal

36. Rowland BD, Bernards R. (2006) Re-evaluating cell-cycle regulation by E2Fs. Cell 127: 871-874.

Figure 1. Pan-cancer expression analysis for DNMT1. (A) The expression of 518 DNMT1 in 33 types of human cancer based on TCGA cancer and normal data. (B-M) 519 DNMT1 expression in TCGA UCEC (B), LIHC (C), BRCA (D), ESCA (E), HNSC (F), THCA (G), STAD (H), READ (I), BLCA (J), KIRC (K), LUAD (L), and LUSC (M) 521 tissues compared with paired normal tissues. *: $P$ value $\leq 0.05 ; * *: P$ value $\leq 0.01 ; * * *$ : $522 \quad P$ value $\leq 0.001$; ns: $P$ value $>0.05$. 

(E), LUSC (F), THCA (G), and LUAD (H). carcinoma

Figure 3. RFS analysis of dichotomized DNMT1 expression in various human cancers. (A-H) The RFS plot of DNMT1 in UCEC (A), BLCA (B), BRCA (C), HNSC (D), LIHC (E), LUSC (F), THCA (G), and LUAD (H). carcinoma; LIHC, liver hepatocellular cancer; BRCA, breast cancer; ESCA, esophageal carcinoma; HNSC, head and neck squamous cell carcinoma; LUSC, lung squamous cell carcinoma; THCA, thyroid carcinoma; BLCA, bladder cancer; LUAD, lung adenocarcinoma

547 DNMT1 in HCC. (A)The miRNA-DNMT1 regulatory network established by 548 Cytoscape software. (B) The expression correlation between predicted miRNAs and 
DNMT1 in HCC analyzed by the starBase database. (C) The expression of hsa-miR-

550 148a-3p in HCC and normal samples in TCGA-LIHC. (D) The prognostic value of hsa-

551 miR-148a-3p in HCC.

552 miRNA, micro RNA; DNMT1, DNA-methyltransferase 1; HCC, hepatocellular carcinoma; LIHC, liver

553 hepatocellular carcinoma; TCGA, The Cancer Genome Atlas

554 Figure 5. Identification of SNHG3 as a potential upstream IncRNAs of hsa-miR-

555 148a-3p in HCC. (A) The lncRNA-mir-148a-3p regulatory network established by

556 Cytoscape software. (B) Venn diagram showing overlaps of 45 possible upstream 557 IncRNAs of mir-148a-3p (LIST2) with DEGs between TCGA-LIHC tumor samples 558 and normal tissues. (C) Volcano plot of differentially expressed lncRNAs (|log2fold 559 change $\mid \geq 1$ and adjusted $P$ value $<0.05$ ). Red represents upregulated genes, and blue 560 indicates downregulated genes. The 3 overlapped genes are highlighted and labeled. (D)

561 The prognostic value of SNHG3 in HCC. (E) The correlation of hsa-miR-148a-3p 562 expressions with SNHG3 expressions in HCC from the starBase v. 3.0 project. (F) The 563 correlation of SNHG3 expressions with DNMT1 expressions in HCC from the starBase 564 v. 3.0 project. (G-I) The expression of DNMT1, miR-148a-3p, and SNHG3 in normal 565 and HCC tissues of different histologic grades. $* P$ value $<0.05$; $* * P$ value $<0.01 ; * * * P$ 566 value $<0.001$.

567 DNA-methyltransferase 1; ncRNA, long non-coding RNA; HCC, hepatocellular carcinoma; DEGs, 568 differentially expressed genes; TCGA-LIHC, The Cancer Genome Atlas liver hepatocellular carcinoma 
570 (A)The level of immune cell infiltration in HCC under different copy numbers of

571 DNMT1. (B-G) The relationship of DNMT1 expression level in HCC with (B ) B cell,

572 (C) CD8+ T cell, (D) CD4+ T cell, (E) macrophage, (F) neutrophil, or (G) DC

573 infiltration level.

574 DNA-methyltransferase 1; HCC, hepatocellular carcinoma; DC, dendritic cell.

575 Figure 7. Relationship between the expression of DNMT1 and PD-1, PD-L1, or

576 CTLA-4 in HCC. (A) The expression correlation of DNMT1 with CTLA-4 in HCC.

577 (B) Spearman's correlation between the expression of DNMT1 and CTLA-4 in HCC

578 adjusted for tumor purity using TIMER. (C) The expression correlation of DNMT1

579 with PDCD1 in HCC. (D) Spearman's correlation between the expression of DNMT1

580 and PDCD1 in HCC adjusted for tumor purity using TIMER. (E) The expression

581 correlation of DNMT1 with CD274 (PD-L1) in HCC. (F) Spearman's correlation

582 between the expression of DNMT1 and CD274 (PD-L1) in HCC adjusted for tumor 583 purity using TIMER.

584 DNA-methyltransferase 1; PD1, programmed cell death protein 1; PD-L1, programmed death ligand 1;

585 HCC, hepatocellular carcinoma; CTLA, cytotoxic T lymphocyte antigen 4.

586 Figure 8. GSEA revealed Hallmark pathways related to DNMT1 in HCC.

587 A. GSEA results showing differential enrichment of genes in Hallmark with high 588 DNMT1 expression. B. GSEA results showing differential enrichment of genes in 589 HALLMARK with low DNMT1 expression. C. The expression correlation of DNMT1 
590 with CDK1 in HCC. D-K. The expression correlation of DNMT1 with E2Fs in HCC:

591 E2F1 (D), E2F2 (E), E2F3 (F), E2F4 (G), E2F5 (H), E2F6 (I), E2F7 (G), and E2F8 (K).

592 DNA-methyltransferase 1; GSEA, Gene Set Enrichment Analysis; HCC, hepatocellular carcinoma.

593 Figure S1. Immune cell score heat map. Different colors represent the expression

594 trend in HCC tissues, and the vertical axis represents the gene expression distribution,

595 where different colors represent different groups. Asterisks represent levels of

596 significance $(* P<0.05, * * P<0.01, * * * P<0.001)$.

597 HCC, hepatocellular carcinoma

598 Table 1. Relationship between DNMT1 expression and clinical characteristics in

599 HCC

600 Table 2. Correlation analysis between DNMT1 and biomarkers of immune cells in

601 HCC determined by the GEPIA database. 
A
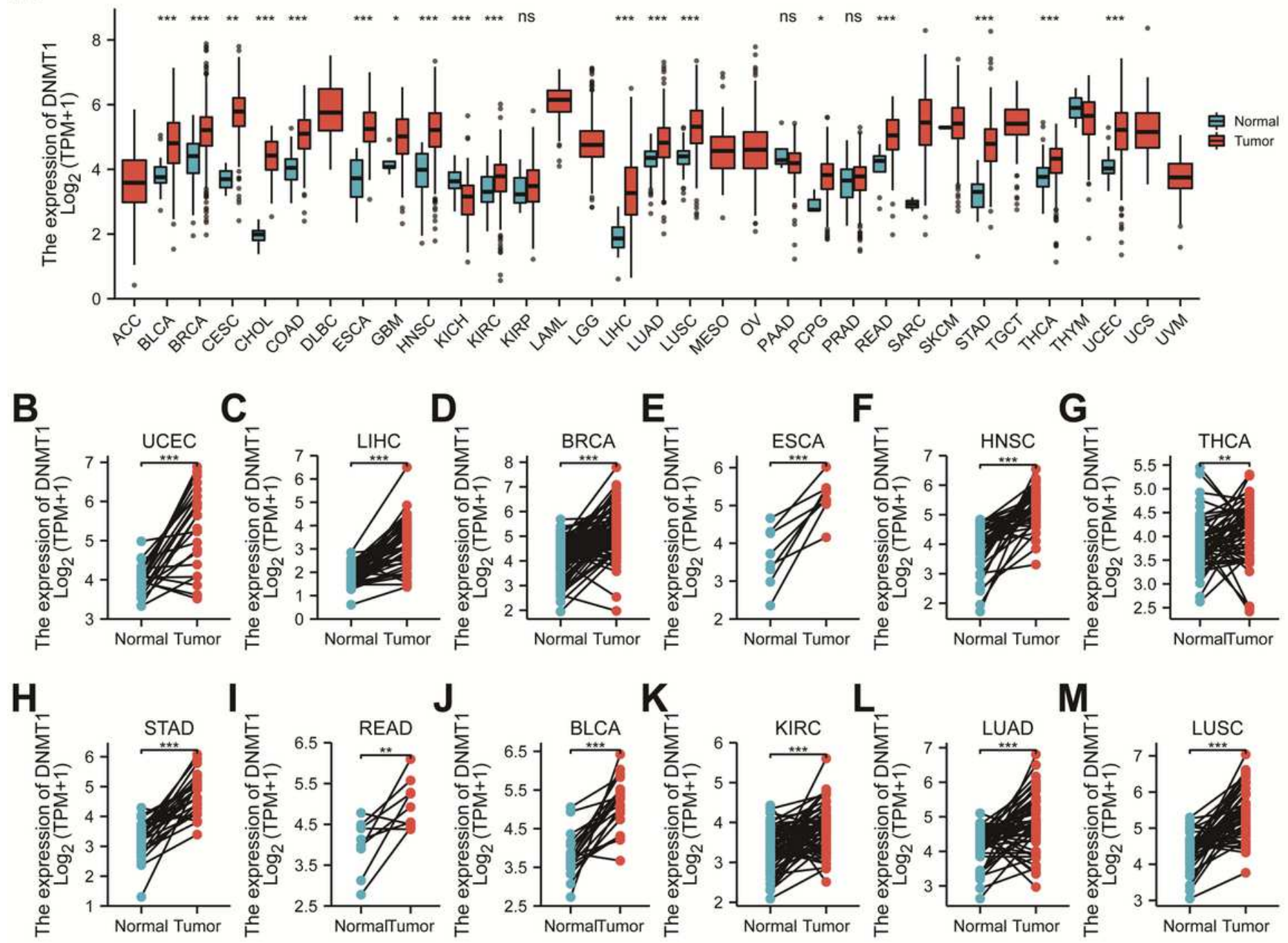

\section{Figure 1}

Pan-cancer expression analysis for DNMT1. (A) The expression of DNMT1 in 33 types of human cancer based on TCGA cancer and normal data. (B-M) DNMT1 expression in TCGA UCEC (B), LIHC (C), BRCA (D), ESCA (E), HNSC (F), THCA (G), STAD (H), READ (I), BLCA (J), KIRC (K), LUAD (L), and LUSC (M) tissues compared with paired normal tissues. *: $P$ value $\leq 0.05$; $* *$ : $P$ value $\leq 0.01$; $* \star \star: P$ value $\leq 0.001$; ns: $P$ value $>0.05$. DNMT1, DNA-methyltransferase 1; TCGA, The Cancer Genome Atlas; UCEC, uterine corpus endometrial carcinoma; LIHC, liver hepatocellular cancer; BRCA, breast cancer; ESCA, esophageal carcinoma; HNSC, head and neck squamous cell carcinoma; THCA, thyroid carcinoma; STAD, stomach adenocarcinoma; READ, rectum adenocarcinoma; BLCA, bladder cancer; KIRC, kidney renal clear cell carcinoma; LUAD, lung adenocarcinoma; KIRC, LUSC, lung squamous cell carcinoma 
A

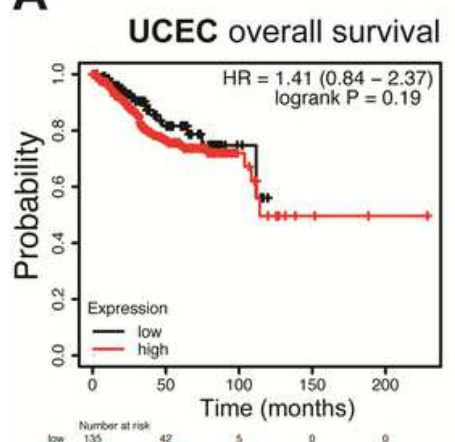

E

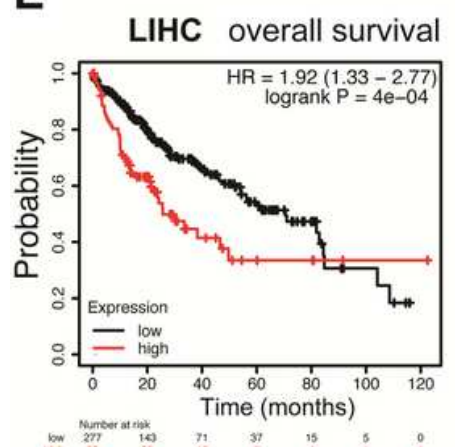

B

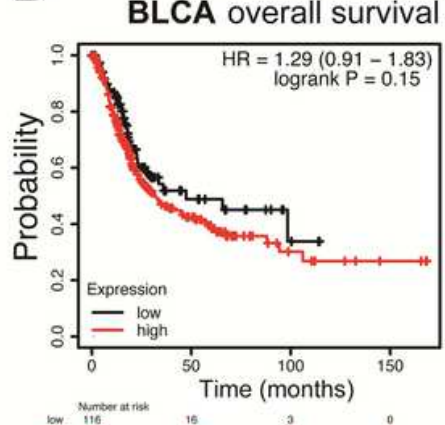

F

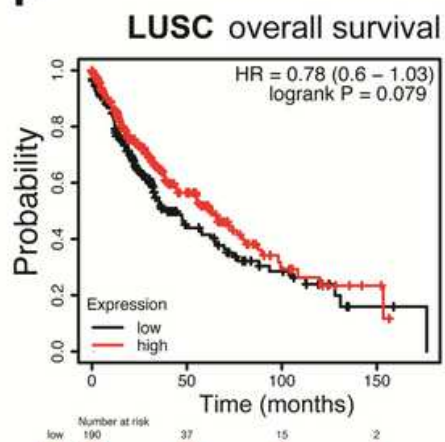

C

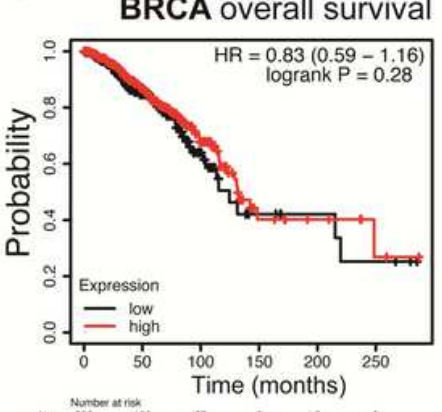

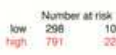

G

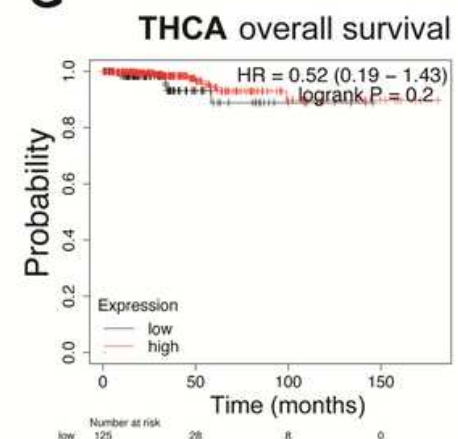

D

HNSC overall survival

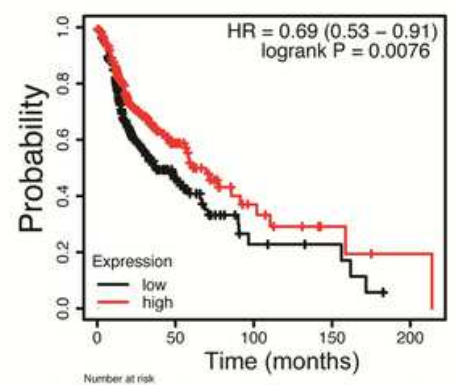

H

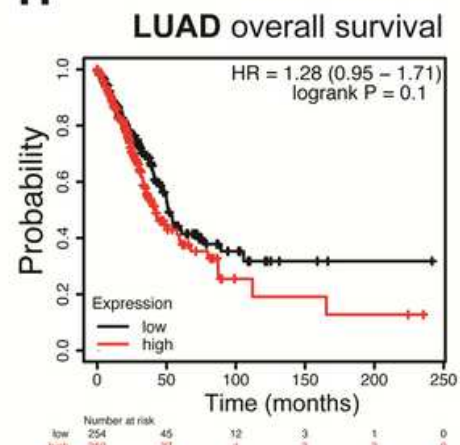

Figure 2

OS of dichotomized DNMT1 expression in various human cancers. $(A-H)$ The OS plot of DNMT1 in $\operatorname{UCEC}(A), B L C A(B), B R C A(C), \operatorname{HNSC}(D), \operatorname{LIHC}(E)$, LUSC (F), THCA (G), and LUAD (H). OS, overall survival; DNMT1, DNA-methyltransferase 1; TCGA, The Cancer Genome Atlas; UCEC, uterine corpus endometrial carcinoma; LIHC, liver hepatocellular cancer; BRCA, breast cancer; ESCA, esophageal carcinoma; HNSC, head and neck squamous cell carcinoma; THCA, thyroid carcinoma; STAD, stomach adenocarcinoma; READ, rectum adenocarcinoma; BLCA, bladder cancer; KIRC, kidney renal clear cell carcinoma; LUAD, lung adenocarcinoma; KIRC, LUSC, lung squamous cell carcinoma 
A

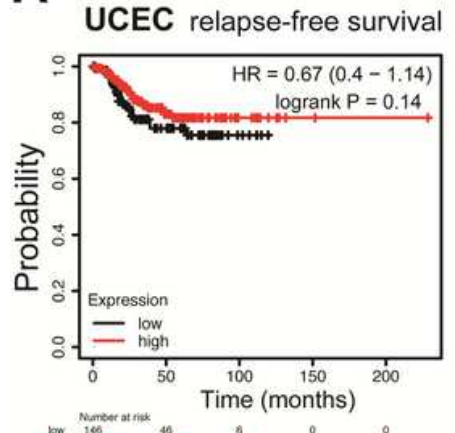

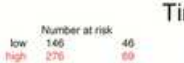

E

LIHC relapse-free survival

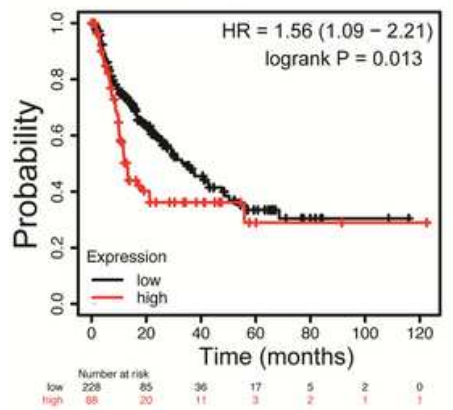

B

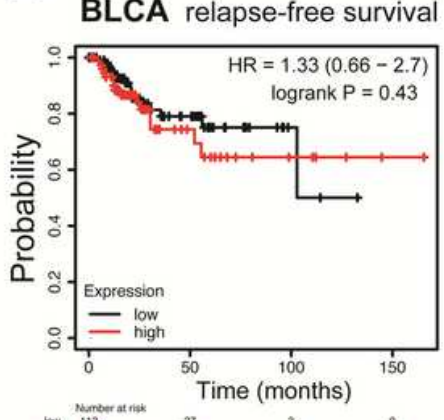

F

LUSC relapse-free survival

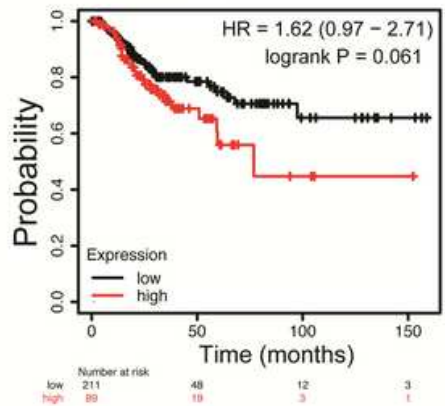

C

BRCA relapse-free survival

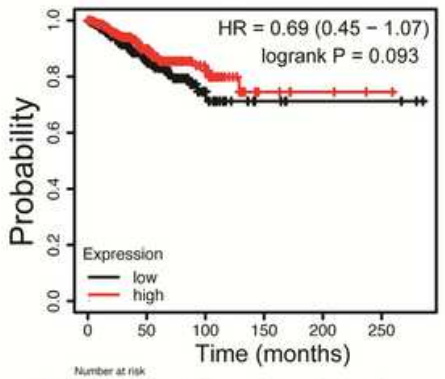

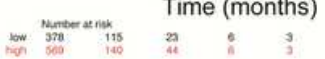

G

THCA relapse-free survival

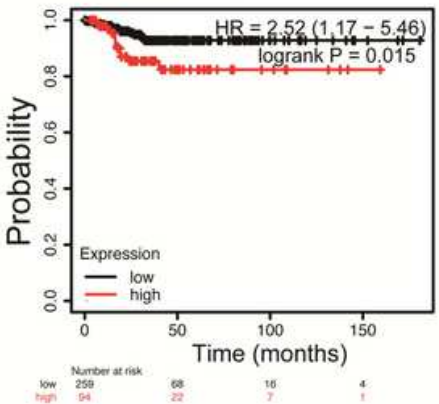

D

HNSC relapse-free survival

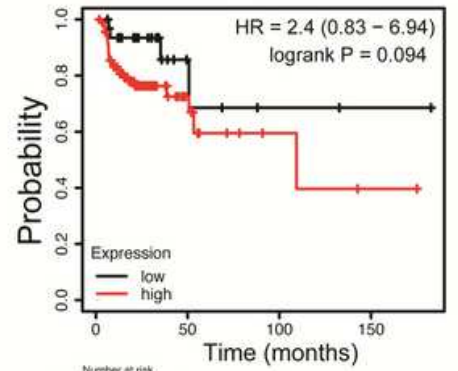

$\mathrm{H}$

LUAD relapse-free survival

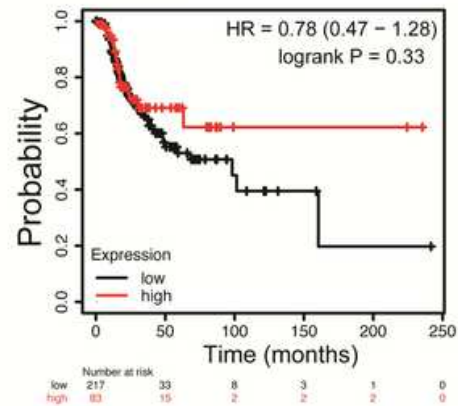

\section{Figure 3}

RFS analysis of dichotomized DNMT1 expression in various human cancers. (A-H) The RFS plot of DNMT1 in UCEC (A), BLCA (B), BRCA (C), HNSC (D), LIHC (E), LUSC (F), THCA (G), and LUAD (H). RFS, relapse-free survival; DNMT1, DNA-methyltransferase 1; UCEC, uterine corpus endometrial carcinoma; LIHC, liver hepatocellular cancer; BRCA, breast cancer; ESCA, esophageal carcinoma; HNSC, head and neck squamous cell carcinoma; LUSC, lung squamous cell carcinoma; THCA, thyroid carcinoma; BLCA, bladder cancer; LUAD, lung adenocarcinoma 


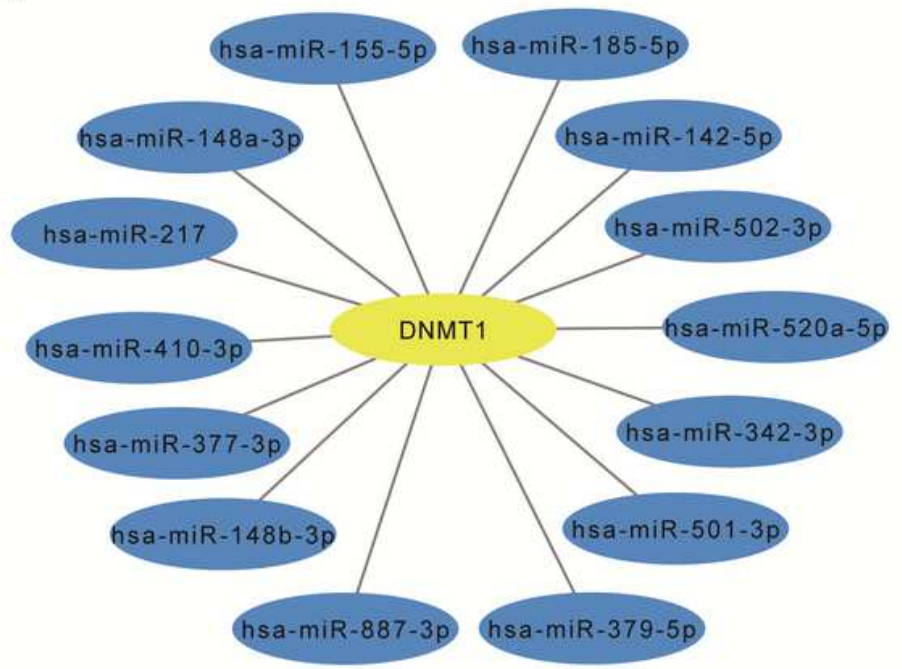

\begin{tabular}{|c|c|c|c|}
\hline Gene & MiRNA & $R$-value & $P$-value \\
\hline DNMT1 & hsa-miR-148a-3p & -0.224 & 1.37E-05 \\
\hline DNMT1 & hsa-miR-148b-3p & 0.331 & $3.38 \mathrm{E}-14$ \\
\hline DNMT1 & hsa-miR-185-5p & 0.142 & $6.18 \mathrm{E}-03$ \\
\hline DNMT1 & hsa-miR-342-3p & 0.181 & 4.73E-04 \\
\hline DNMT1 & hsa-miR-887-3p & 0.201 & 9.93E-05 \\
\hline DNMT1 & hsa-miR-379-5p & 0.236 & 4.52E-06 \\
\hline DNMT1 & hsa-miR-217 & 0.136 & 8.92E-03 \\
\hline DNMT1 & hsa-miR-142-5p & 0.265 & 2.27E-07 \\
\hline DNMT1 & hsa-miR-502-3p & 0.151 & $3.51 \mathrm{E}-03$ \\
\hline DNMT1 & hsa-miR-377-3p & 0.193 & $1.85 \mathrm{E}-04$ \\
\hline DNMT1 & hsa-miR-520a-5p & 0.154 & 2.93E-03 \\
\hline DNMT1 & hsa-miR-410-3p & 0.209 & $5.22 \mathrm{E}-05$ \\
\hline DNMT1 & hsa-miR-501-3p & 0.237 & 4.00E-06 \\
\hline DNMT1 & hsa-miR-155-5p & 0.255 & 6.86E-07 \\
\hline
\end{tabular}

C

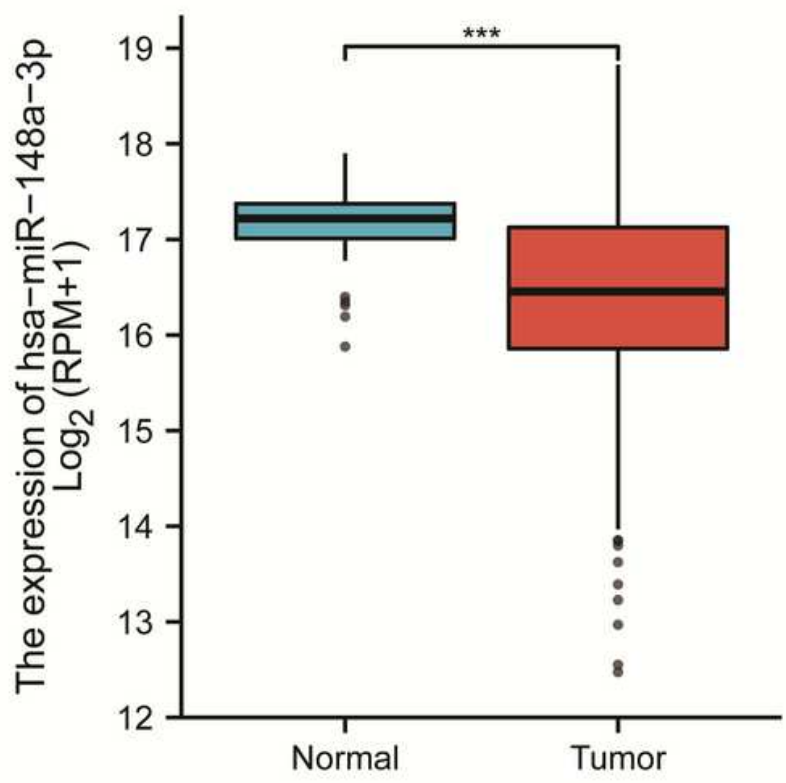

D

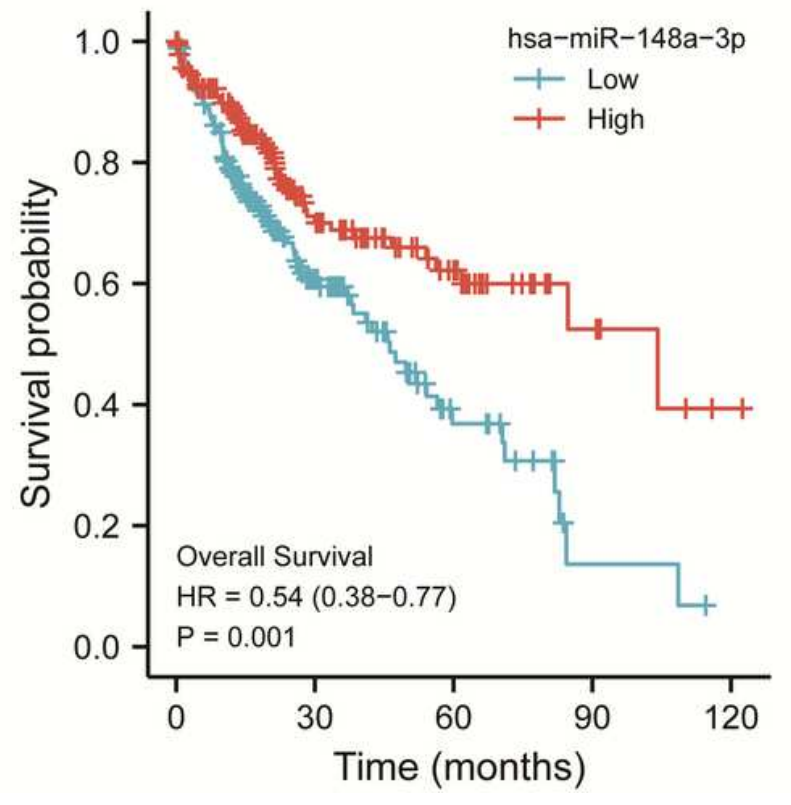

Figure 4

Identification of hsa-miR-148a-3p as a potential upstream miRNA of DNMT1 in HCC. (A)The miRNADNMT1 regulatory network established by Cytoscape software. (B) The expression correlation between predicted miRNAs and DNMT1 in HCC analyzed by the starBase database. (C) The expression of hsamiR-148a-3p in HCC and normal samples in TCGA-LIHC. (D) The prognostic value of hsa-miR-148a-3p in HCC. miRNA, micro RNA; DNMT1, DNA-methyltransferase 1; HCC, hepatocellular carcinoma; LIHC, liver hepatocellular carcinoma; TCGA, The Cancer Genome Atlas 
A

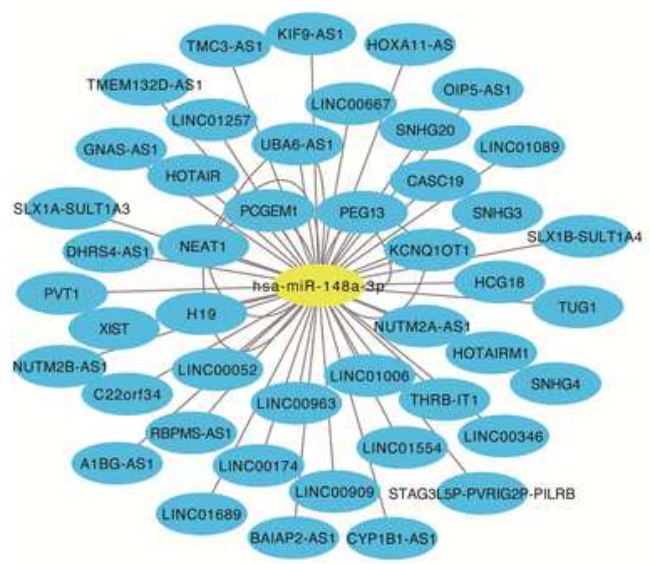

D

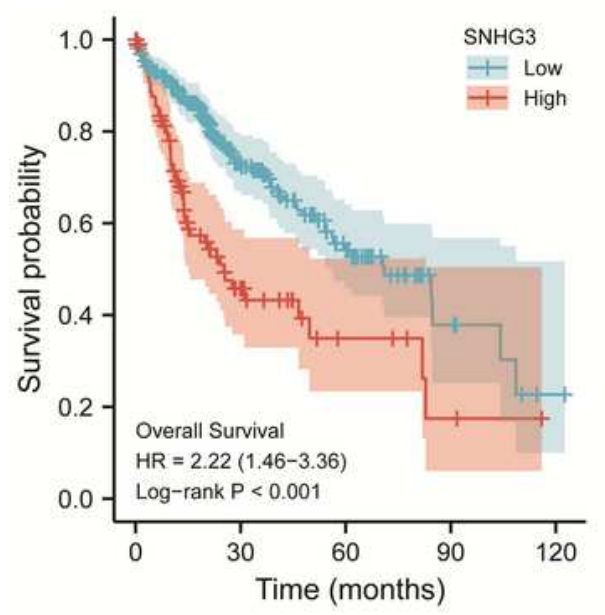

G

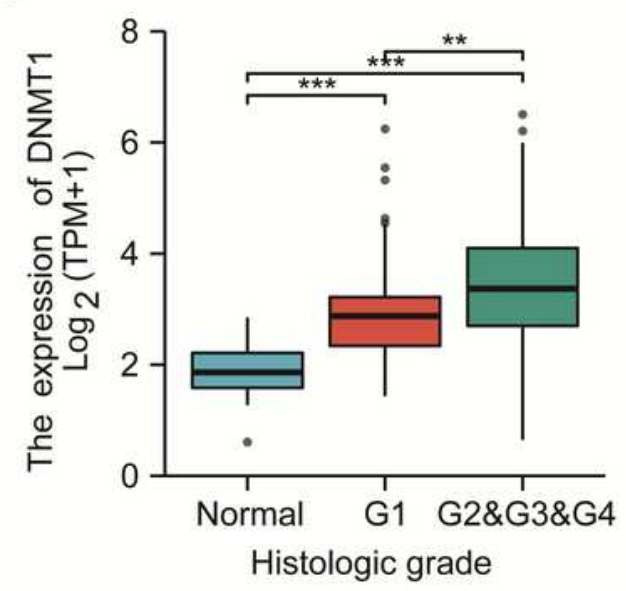

B

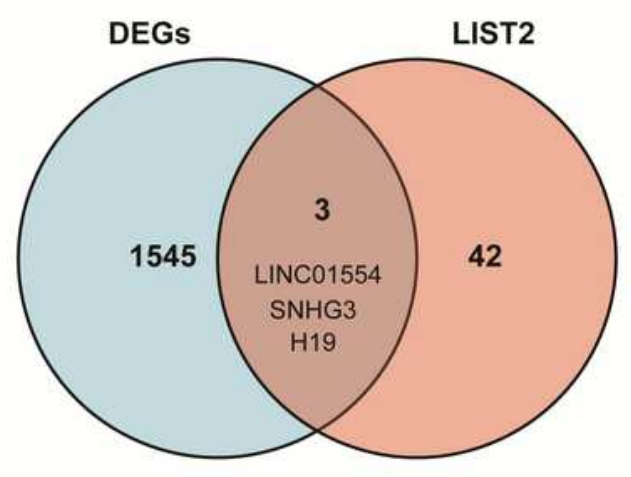

E

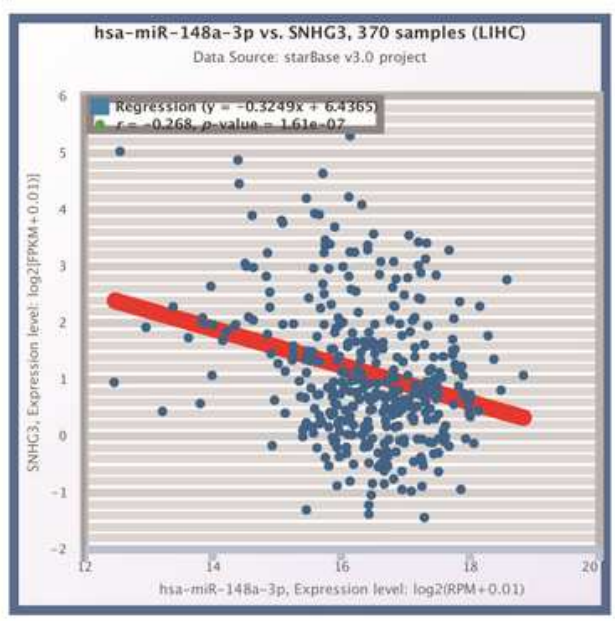

H

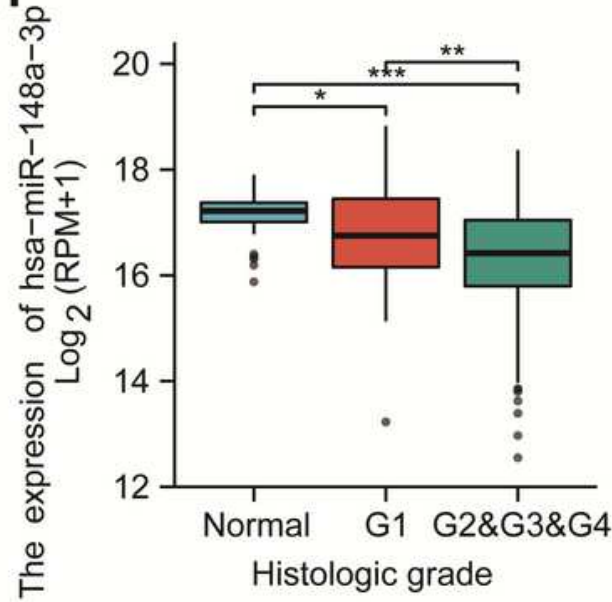

C

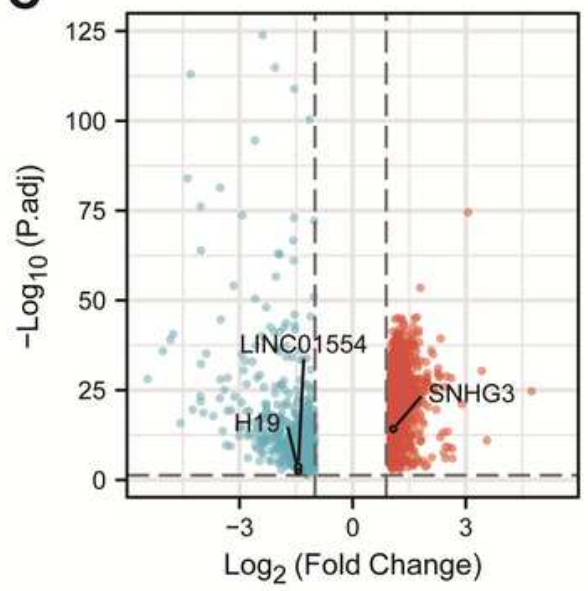

F

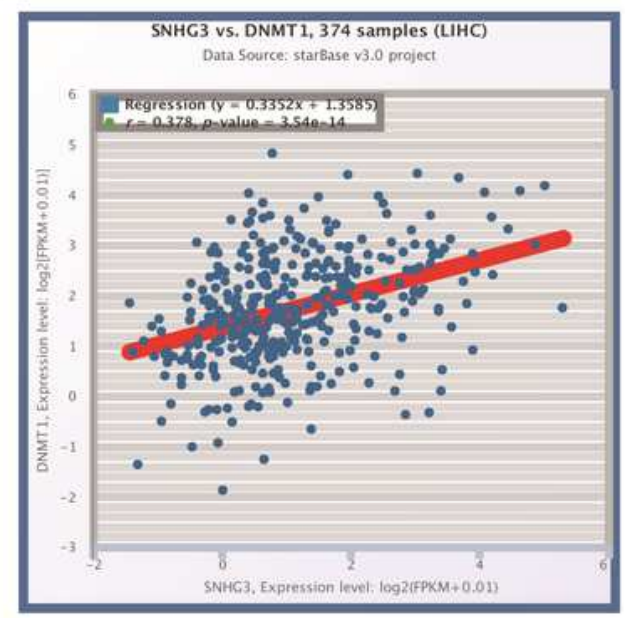

I

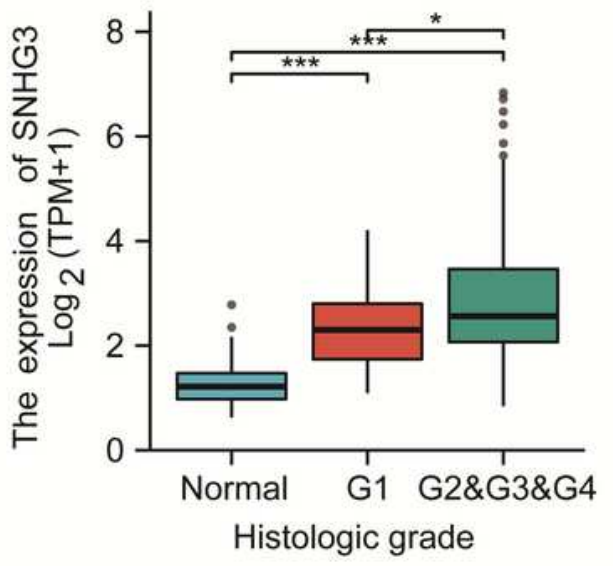

Figure 5

Identification of SNHG3 as a potential upstream IncRNAs of hsa-miR-148a-3p in HCC. (A) The IncRNAmir-148a-3p regulatory network established by Cytoscape software. (B) Venn diagram showing overlaps of 45 possible upstream IncRNAs of mir-148a-3p (LIST2) with DEGs between TCGA-LIHC tumor samples and normal tissues. (C) Volcano plot of differentially expressed IncRNAs (|log2fold changel $\geq 1$ and adjusted $P$ value $<0.05)$. Red represents upregulated genes, and blue indicates downregulated genes. The 
3 overlapped genes are highlighted and labeled. (D) The prognostic value of SNHG3 in HCC. (E) The correlation of hsa-miR-148a-3p expressions with SNHG3 expressions in HCC from the starBase v. 3.0 project. (F) The correlation of SNHG3 expressions with DNMT1 expressions in HCC from the starBase v. 3.0 project. (G-I) The expression of DNMT1, miR-148a-3p, and SNHG3 in normal and HCC tissues of different histologic grades. ${ }^{*}$ value $<0.05$; $* * P$ value $<0.01$; $* \star * P$ value $<0.001$. DNA-methyltransferase 1 ; ncRNA, long non-coding RNA; HCC, hepatocellular carcinoma; DEGs, differentially expressed genes; TCGA-LIHC, The Cancer Genome Atlas liver hepatocellular carcinoma

A
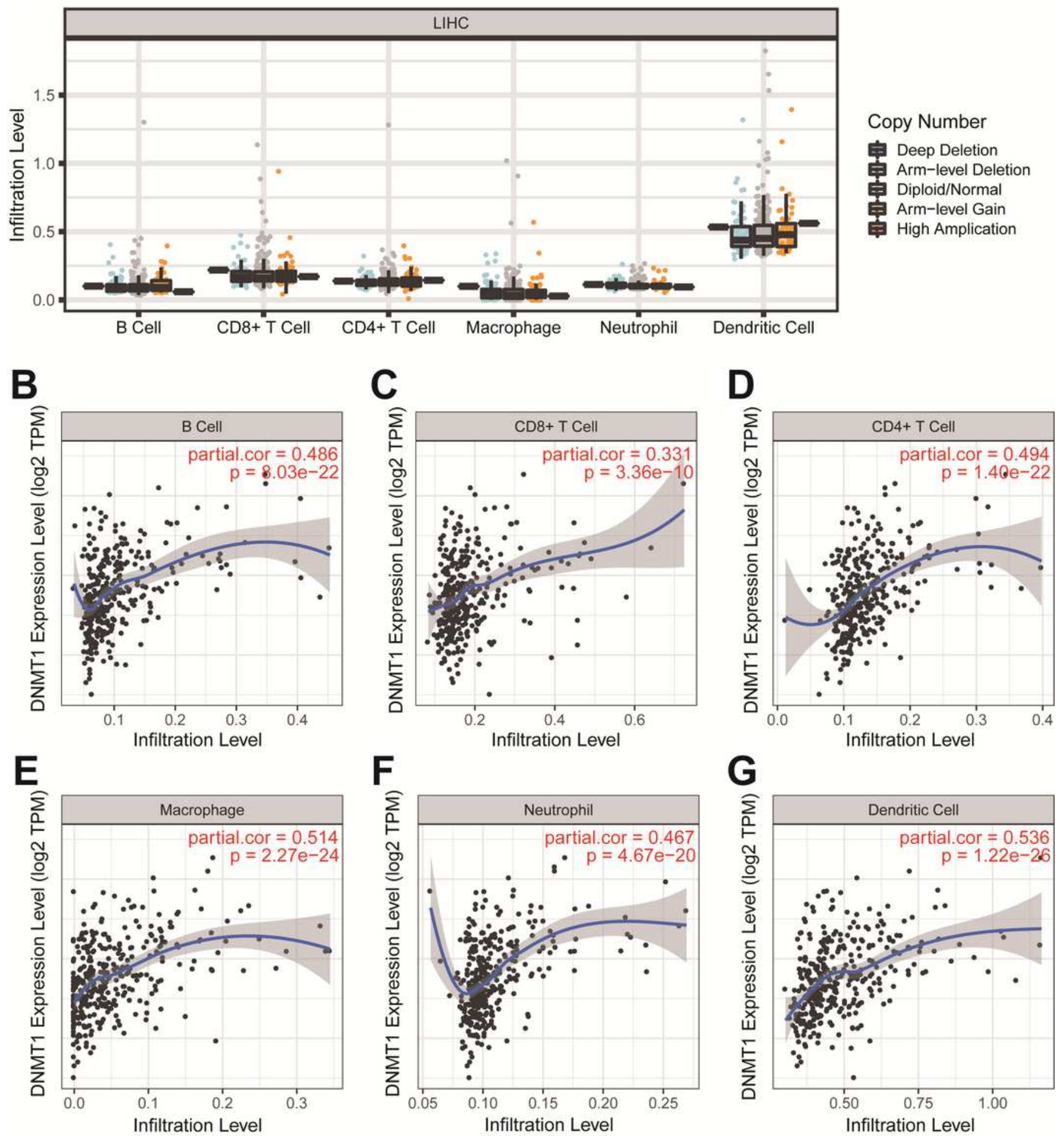
Figure 6

Correlation between DNMT1 levels and immune cell infiltration in HCC. (A)The level of immune cell infiltration in HCC under different copy numbers of DNMT1. (B-G) The relationship of DNMT1 expression level in HCC with (B ) B cell, (C) CD8+ T cell, (D) CD4+ T cell, (E) macrophage, (F) neutrophil, or (G) DC infiltration level. DNA-methyltransferase 1; HCC, hepatocellular carcinoma; DC, dendritic cell.

A

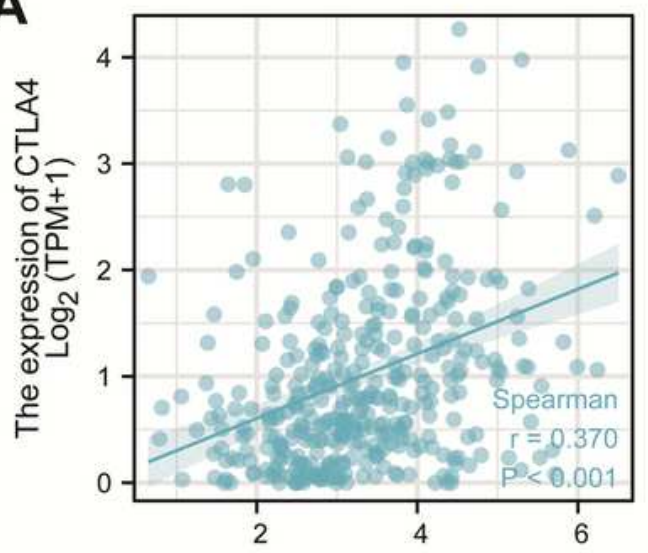

The expression of DNMT1 $\log _{2}(T P M+1)$

C

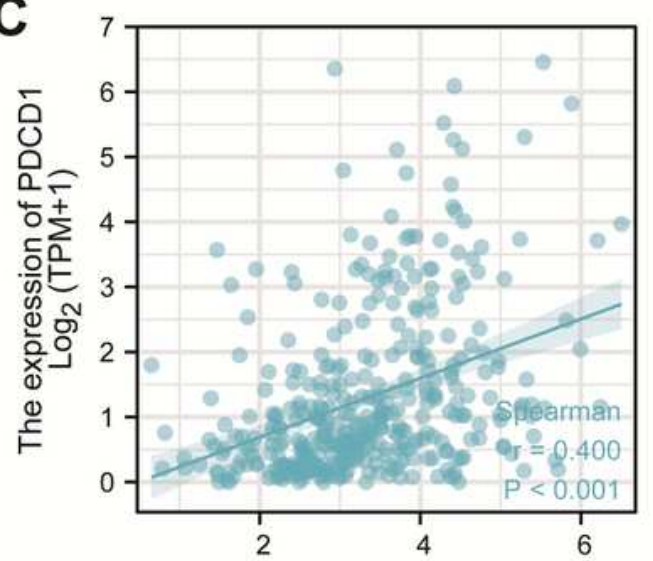

The expression of DNMT1 $\log _{2}(\mathrm{TPM}+1)$

E

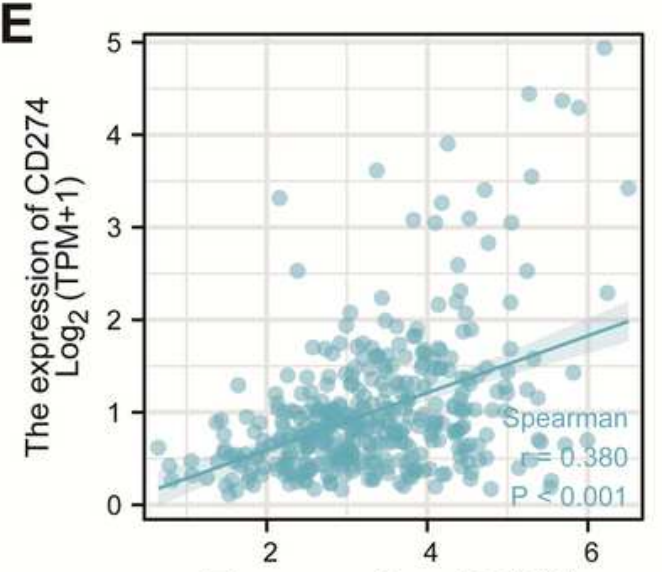

The expression of DNMT1 $\log _{2}(T P M+1)$
B

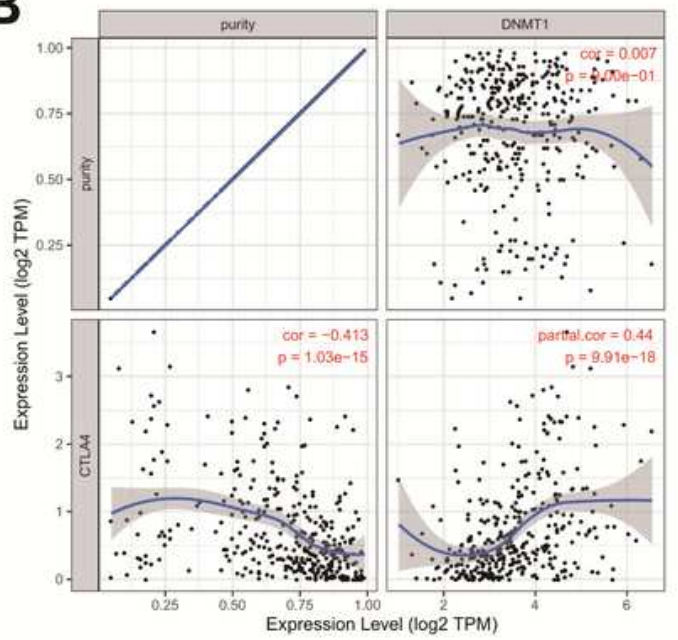

D

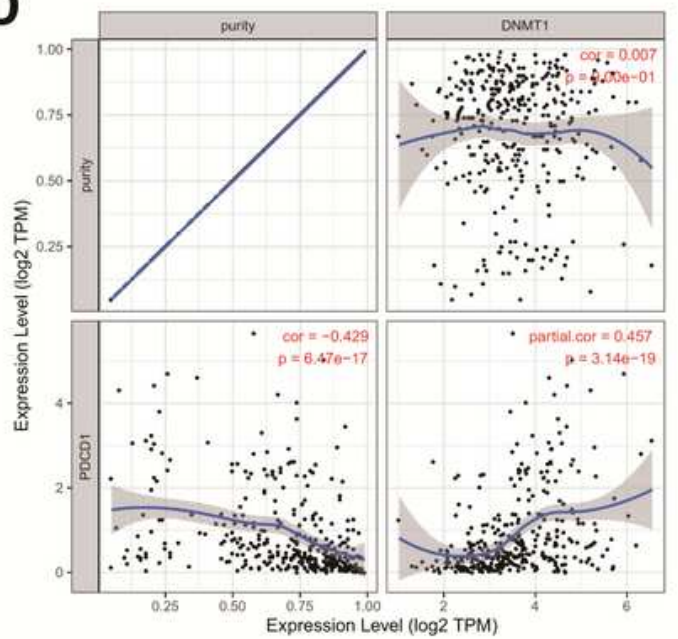

F

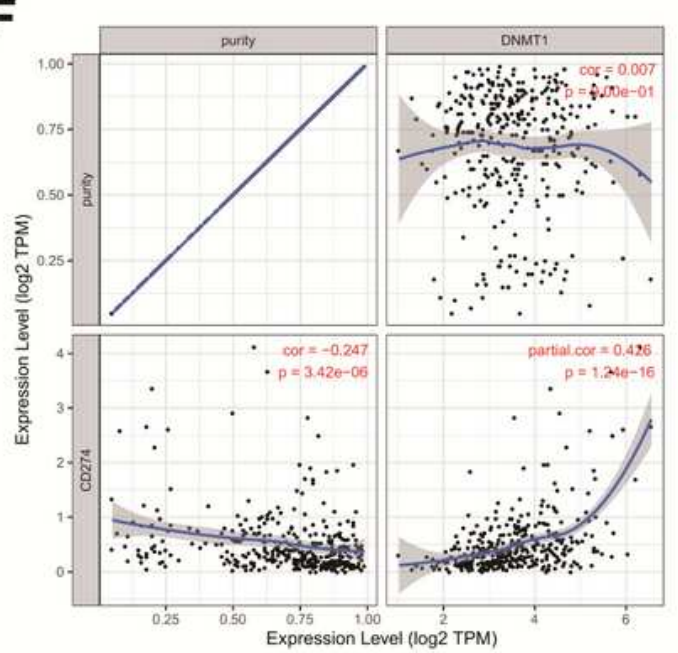


Relationship between the expression of DNMT1 and PD-1, PD-L1, or CTLA-4 in HCC. (A) The expression correlation of DNMT1 with CTLA-4 in HCC. (B) Spearman's correlation between the expression of DNMT1 and CTLA-4 in HCC adjusted for tumor purity using TIMER. (C) The expression correlation of DNMT1 with PDCD1 in HCC. (D) Spearman's correlation between the expression of DNMT1 and PDCD1 in HCC adjusted for tumor purity using TIMER. (E) The expression correlation of DNMT1 with CD274 (PD-L1) in HCC. (F) Spearman's correlation between the expression of DNMT1 and CD274 (PD-L1) in HCC adjusted for tumor purity using TIMER. DNA-methyltransferase 1; PD1, programmed cell death protein 1; PD-L1, programmed death ligand 1; HCC, hepatocellular carcinoma; CTLA, cytotoxic T lymphocyte antigen 4.

A

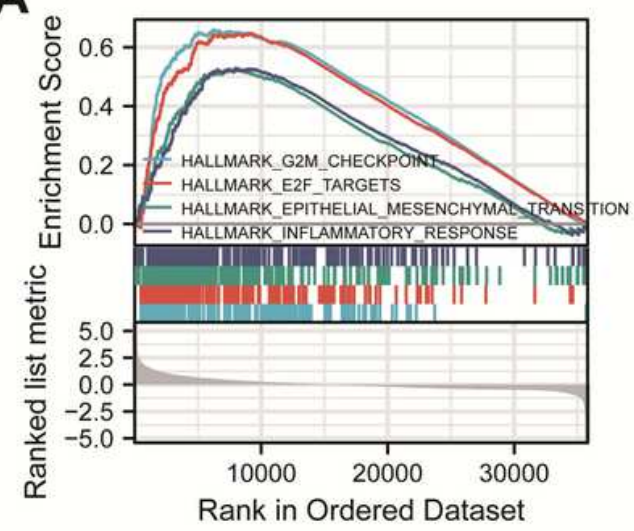

D

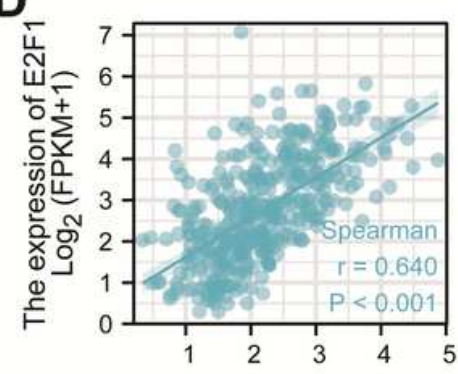

The expression of DNMT1 $\log _{2}(\mathrm{FPKM}+1)$

$\mathrm{H}$

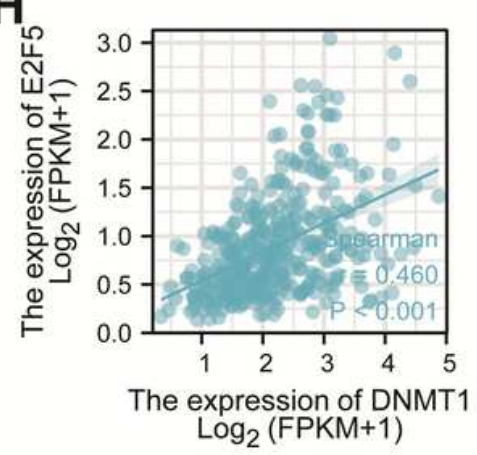

E
B
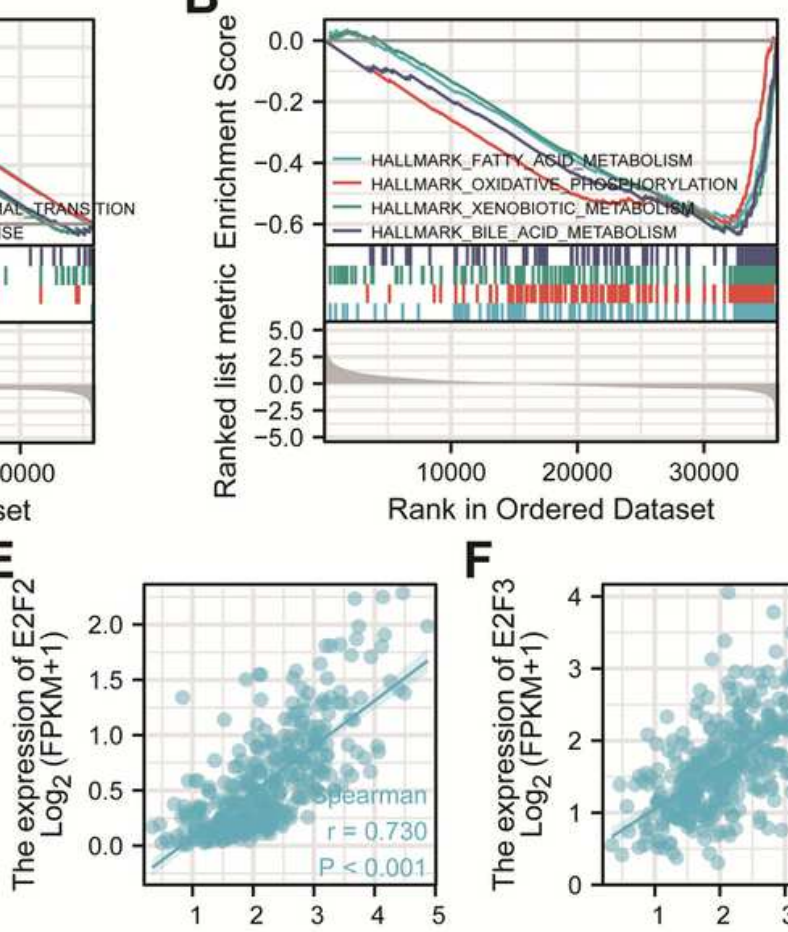

The expression of DNMT1 $\log _{2}(\mathrm{FPKM}+1)$

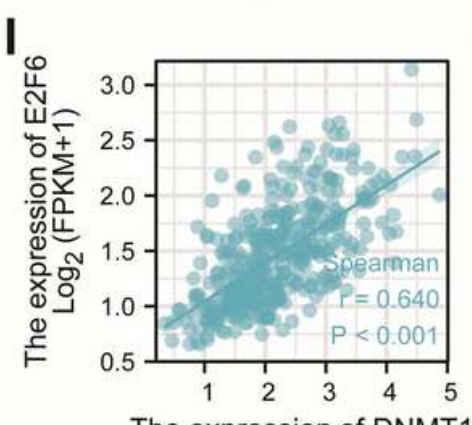

The expression of DNMT1 $\log _{2}(\mathrm{FPKM}+1)$

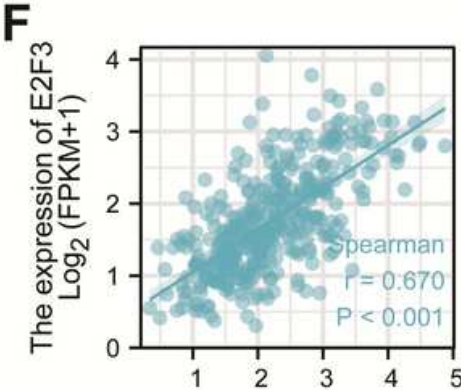

The expression of DNMT1 $\log _{2}(\mathrm{FPKM}+1)$

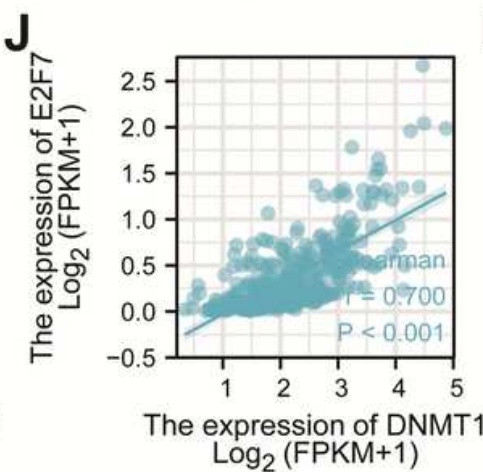

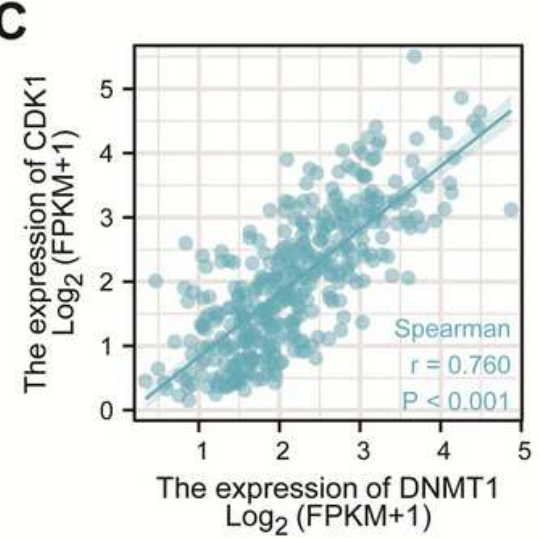

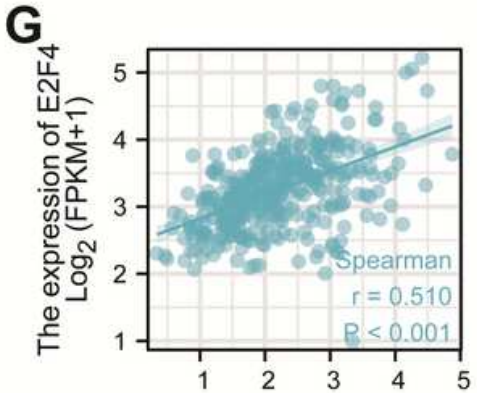

The expression of DNMT1 $\log _{2}(\mathrm{FPKM}+1)$

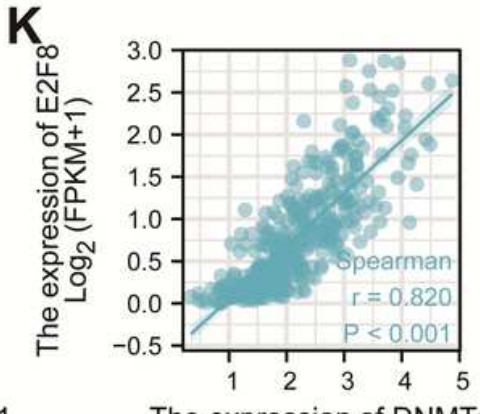

The expression of DNMT1 $\log _{2}(\mathrm{FPKM}+1)$

\section{Figure 8}

GSEA revealed Hallmark pathways related to DNMT1 in HCC. A囚GSEA results showing differential enrichment of genes in Hallmark with high DNMT1 expression. B囚GSEA results showing differential enrichment of genes in HALLMARK with low DNMT1 expression. C $\triangle$ The expression correlation of DNMT1 with CDK1 in HCC. D-K. The expression correlation of DNMT1 with E2Fs in HCC: E2F1 (D), E2F2 (E), E2F3 
(F), E2F4 (G), E2F5 (H), E2F6 (I), E2F7 (G), and E2F8 (K). DNA-methyltransferase 1; GSEA, Gene Set Enrichment Analysis; $\mathrm{HCC}$, hepatocellular carcinoma.

\section{Supplementary Files}

This is a list of supplementary files associated with this preprint. Click to download.

- Table1.pdf

- Table2.pdf

- FigS1.tif 